\title{
Inter-individual variability in gut microbial metabolism and vascular response to lignans and isoflavones: the StratiPol study
}

\author{
W. Alotaibi, P.Young Tie Yang, W.L. Hall and A. Rodriguez-Mateos \\ Department of Nutritional Sciences, School of Life Course Sciences, Faculty of Life Sciences and Medicine, King's \\ College London, UK
}

Objectives: Evidence shows inter-individual variability in gut microbial metabolism of phytoestrogens, lignans and isoflavones, but little is known on whether this is associated with health benefits ${ }^{(1)}$. The aim of this project is to investigate the variability in gut microbial metabolism and vascular response to lignans \& isoflavones in a group of healthy individuals.

An open-label, single-arm study investigating the influence of gut microbial metabolism variability on vascular response to a (poly) phenol rich breakfast is currently ongoing in a group of healthy men and women aged $38 \pm 13$ years old and with BMI $24 \pm 3 \mathrm{~kg} / \mathrm{m}^{2}$. Participants consumed a (poly)phenol rich breakfast containing $300 \mathrm{mg}$ of flaxseed lignans and $22 \mathrm{mg}$ of soy milk isoflavones for 3 days. Urine samples were collected for 24 hours after consumption on day 4 . Flow mediated dilation, pulse wave velocity, augmentation index, blood pressure, heart rate and serum biochemistry were assessed at baseline after an overnight fast on day 1 and $24 \mathrm{~h}$ after the last breakfast was consumed on day 4. Urine samples were analysed using liquid chromatography tandem mass spectrometry (LC-MS/MS) and authentic standards.

Preliminary results from the first 69 volunteers (48 females vs 21 males) are presented. The main metabolites of the isoflavone daidzin (S-equol, daidzein, R,S equol 4'-sulfate, and R,S equol 7- $\beta$-D-glucuronide), and the lignan, secoisolariciresinol diglucoside $(( \pm)$-enterolactone, enterodiol, $( \pm)$-Enterolactone monosulfate and $( \pm)$-Enterolactone mono- $\beta$-D-glucuronide) gut microbial metabolites were identified and quantified in $24 \mathrm{~h}$ urine. In this population, $74 \%$ of the participants were non-equol producers $(31 \%$ male vs $69 \%$ female) and $26 \%$ were equol producers (28\% male vs $72 \%$ female). A significant decrease in systolic and diastolic blood pressure was found after 3 days isoflavone consumption only in male equol producers $(120 \pm 10$ to $113 \pm 8 \mathrm{mmHg}, \mathrm{p}=0.0046$ and $77 \pm 11$ to $71 \pm 12 \mathrm{mmHg}, \mathrm{p}=0.0024$ respectively). All volunteers produced enterolactone metabolites, with a high variability in urinary levels of total and individual enterolactone metabolites. No significant differences in any vascular outcome were found between low (lower quartile $\leq 10 \mu \mathrm{mol}, 29 \%$ men and $71 \%$ female) and high (higher quartile $\geq 44 \mu \mathrm{mol}, 35 \%$ men and $65 \%$ female) enterolactone producers.

Sex and gut microbiota may be important factors explaining variability in the vascular response to isoflavones. More research is needed to confirm these findings, including randomised controlled trials with previous stratification by sex and polyphenol gut microbial metabotype.

\section{Acknowledgements}

The authors acknowledge a Kings Faisal University (Saudi Arabia) PhD scholarship to Wafa Alotaibi.

\section{Reference}

1. Cortés-Martín A, Selma MV, Tomás-Barberán FA et al. (2020) Mol Nutr Food Res 64, e1900952. 